\title{
Workers' postural conditions in the charcoal production proccess based on vertical metallic cylynders
}

\author{
Ivana Márcia Oliveira Maia, ${ }^{\mathrm{a},}$ and Antonio Carlos de Francisco ${ }^{\mathrm{b}}$ \\ ${ }^{a}$ Federal Institute of Education, Science and Technology of Maranhão, Av. Getúlio Vargas, $n^{\circ} 04$ - Monte Castelo \\ - São Luís-MA, Brazil \\ ${ }^{b}$ Departamento de Pós-Graduação Pitangüi, Technological Federal University of Paraná, Av. Monteiro Lobato, \\ Km 04 - 84016-210 - Ponta Grossa, PR - Brasil
}

\begin{abstract}
Considering the importance of posture to the workers' health in the production of charcoal, this paper presents an ergonomic research based on a biomechanical focus that aims to evaluate the posture adopted by these workers on the production of charcoal in vertical metallic cylinders. Thus, it was verified the incidence of pain and/or musculoskeletal injuries to these workers. Also, it was evaluated the weight carried by them and the positions taken in their daily tasks. Applying the Ergonomic Analysis of Labor, the data collection was done by directly observing the workers, registering images, by interviews, and posture analysis based on the OWAS method. The main results of the research show that there are postures with risks in the four levels of musculoskeletal injuries classified by OWAS, concluding that the method is imperative for ergonomic recommendations for minimization or eradication of suffering injury and worker's postural constraints.
\end{abstract}

Keywords: postural condition, charcoal workers, CDM

*Corresponding author. E-mail: ivana-marcia@hotmail.com.. 


\section{Introduction}

In the charcoal production in Brazil, labor is associated to suffering and pain. This occurs due to inadequacy of work conditions and hard nature of the tasks that workers must accomplish, which affect their health and performance.

Brazil is the bigger charcoal producer in the world (Guimarães Neto, 2005). In 2003 Brazil was the first producer and consumer of charcoal in the world, producing 29.202 millions of cubic meters.

Charcoal has high importance in the pig iron production, which is of high relevance to the Brazilian economy. However, the charcoal production is conducted in an irregular way, offending the environment and the citizen rights. For this reason, that this industry is cited as one of the productive segments that most disrespect the works basics rights and cause environment damages in Brazil.

This way, it's of high relevance the contribution to the research and development of a charcoal production method that minimizes the environment damages produced and prevents the health risks applied to the workers, generating a sustainable charcoal production process.

One of the production processes proposed to minimize these troubles is the production of charcoal through carbonization using metallic cylinders, which offers a better use of wood and minimizes the environment damages.

This work presents an evaluation of postural conditions of workers involved in the charcoal production process based on carbonization using vertical metallic cylinders. The evaluation is performed by the postural biomechanics, that accordingly to [2], is based on the evaluation of the posture adopted by the worker associated with the load to which he is submitted. The results indicate that the worker's posture may become a productivity tool if carefully verified.

Additionally, this work presents results obtained from a verification of pain and incidence of musculoskeletal lesions, workers' submission levels and the diagnosis of work conditions related to the posture required to execute the necessary tasks. These are related to the OWAS method. Finally, we present some ergonomic alternatives aiming at the minimization or eradication of workers lumbar sufferings and postural constraints.

This work is organized such that section 2 presents the charcoal production system and the methodology used in the research. Following, section 3 shows the results obtained and discuss them. Finally, section 5 presents final remarks and future research directions.

\section{Methods}

\subsection{The charcoal production}

The Wood carbonization using vertical metallic cylinders is done in the following sequence of tasks: wood discharge, wood supply to the carbonization place; positioning of cylinders for charge; cylinder charge; cylinder closing; cylinder positioning in wait; ignition of the carbonization chamber; cylinder reposition in the stove; Carbonization; verification of carbonization; removal of cylinders from stove; cooling the cylinder; discharge of the cylinder; product selection; positioning the cylinder for discharge; product selection; removal; carbonization place cleaning.

\subsection{Subjects}

In this work we observed the biomechanical performance of three teams of workers from a total of four teams that actually work in the observed site. Each of these teams is composed of four workers, who work in alternate turns and maintain an uninterrupted production. We observed that male workers characterize the population in the research, with ages between 20 and 31 years old, and literate. The workers have a daily work journey of six hours, with 15minutes intervals, after the first three hours, and a 2 hours mealtime. All of them were working for seven months in this activity.

The workers may play two roles in the team: team leader and carbonization operator.

The team leader has to track the stove and cylinders weight, the temperature and carbonization time, and also dispose and remove the cylinders in the stove, remove charcoal from cylinder and load charcoal truck. This team member suffers from mental requirements, executing tasks that are not characterized by lifting load or repetitive motions.

The remaining team members are divided to execute tasks related to the productive process. Among the observed tasks, those that presented more postural requirements were: the metallic cylinder charge, the carbonization verification, the chamber ignition, the charcoal distribution in the deposit and the carbonization place cleanup.

Two workers execute the metallic cylinder charge. These two workers take the cylinders with wood and 
put them in the stove. It was observed and registered the charge of 10 cylinders, with average execution time of 1hour and 5 minutes.

The task of carbonization verification is executed by visually verifying the firewood burn state that maintains the cylinder heating and by moving ignition ember. This task is executed observing through windows $(0.50 \mathrm{~m} \times 0.30 \mathrm{~m})$ located at $0.50 \mathrm{~m}$ from the ground level and inclusion through this window of a tool to move the burning firewood. The worker walks the entire carbonization chamber and flexes the body to see through the windows located in each of the walls of the carbonization chamber. In the observed carbonization place, there were ten carbonization chambers. Each chamber is verified once per carbonization hour.

To accomplish the chamber ignition task, the worker transports the firewood to the chamber, and then igniting the fire. In this task it is used approximately $117 \mathrm{Kg}$ of firewood. The motions from firewood collection until the deposition of the firewood in the carbonization chamber occur in an average time of 23 minutes.

The distribution of charcoal in the depository is executed by distributing the charcoal in the depository silo. To ensure the quality requirements, the worker must move the charcoal over the sieve to separate the unprocessed charcoal. During this procedure the worker walks around the depository, in order to make possible the motion of all the charcoal in the sieve. This task is accomplished in an average time of 7 minutes and 28 seconds, with repetition of the motion in five points. In this task legs and body are not submitted to biomechanical demand, which was observed in the arms.

The carbonization place cleanup removes the solid leavings that result from the previous work turn. The task is performed sweeping the charcoal in the ground, collecting and putting it on the depository sieve. All the workers in the team are in charge of this task, which takes an average time of 15 minutes.

\subsubsection{Procedure}

Ergonomics is a knowledge area that studies the relations between the man and its work. It's not possible to execute an Ergonomic Analysis of Work (EAW) of an entire industrial floor. So, we need to determine a specific focus to generate a recommendation or a report.

The EAW is a tool for strict evaluation, which is used to analyze individual problems. Basically, the EAW is a qualitative evaluation, which is based on a sequence of data and information collection that will be used to produce the necessary changes in the work environment, aiming at the worker's satisfaction.

The data collection was done through the observation and image registration, interviews and application of specific forms. The methods used in this work were: OWAS system [6] e [5], painful area diagram [1], [4] and [5], eletromyogram registration and checklist [2].

\section{Results and discussion}

This section presents the analysis of data collected in an industrial floor that produces charcoal using the metallic cylinders. The results are presented with three approaches. The first one evaluates the results of the analysis of each posture event, from the observation of which tasks has greater biomechanical risk. Following, we present the analysis and discussion of the results based on each method classification category. Finally, the results are discussed according to executed tasks.

Initially, we observed the tasks that present more postural requirements and identified the functions that were associated to lesion risks, pain and musculoskeletal discomfort. This was done by direct observing and by taking photographs/vídeos. In the proceeded evaluation, the Painfull Areas Diagram from Corlett and Manenica did not present a registration of biomechanical unbalance in all of the studied population.

The tasks that, accordingly with the study, present more postural requirements are:

- Cylinder Lifting;

- Chamber Ignition;

- Carbonization Chamber Verification;

- Charcoal selection;

- Carbonization Place Cleanup;

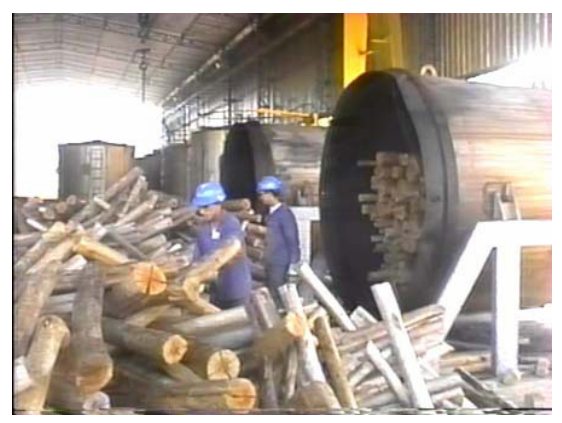

Figure 1 - Cylinder Charge 


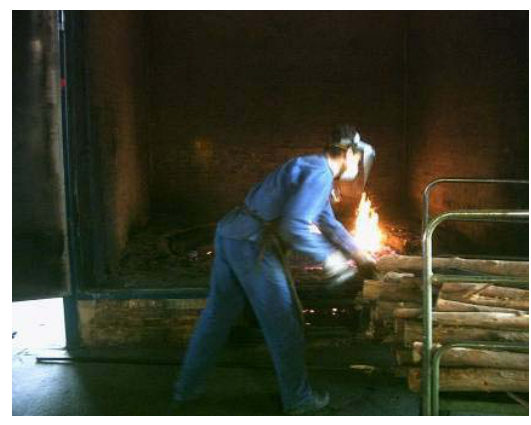

Figure 2 - Chamber Ignition

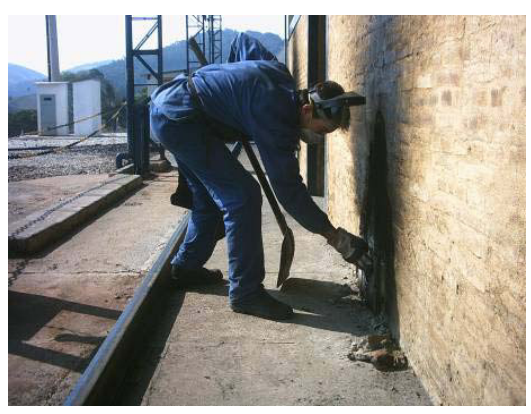

Figura 3 - Chamber Verification

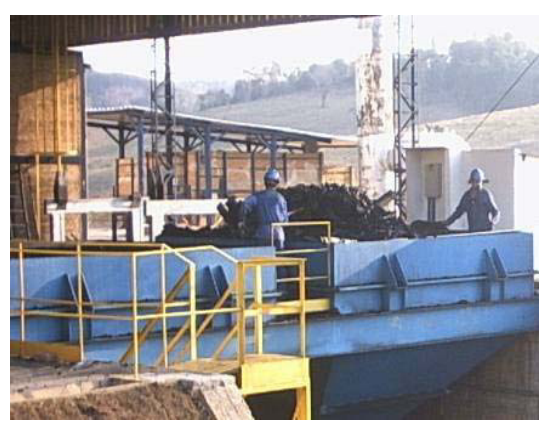

Figure 4-Charcoal Selection

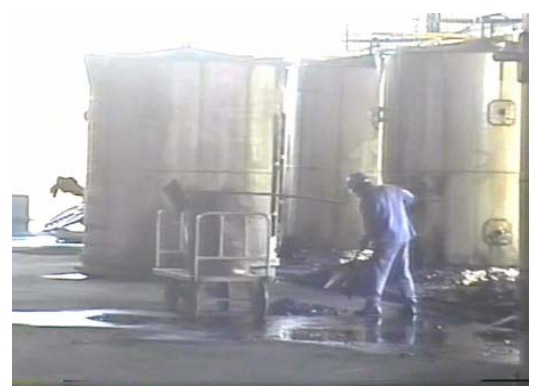

Figura 5 - Carbonization Place Cleanup

\subsection{Biomechanical evaluation of charcoal workers}

The team leader is in charge of ensuring the performance and productivity level of the team. Additionally he monitors: the stove, the cylinder weight relation, the temperature and the carbonization time. In summary, he supervises all tasks. These responsibilities add strong mental requirements.

We observed that the nature of the task requires constant alert state from all the team members, to avoid carbonization above the previously defined time, adequate to the stove, that could cause fire and/or production damage.

The biomechanical leader's work is in general stand up and statically positioned. But, periodically he walks the entire production floor. Some studies like [2], [5] and [7] stated that the alternation between dynamic and static work results in biomechanical equilibrium.

We also observed that the team leader executes tasks that are not related to load lifting or repetitive motions.

The carbonization operators execute several different tasks integrated to the production process. From the observed tasks, the cylinder charge (1), the carbonization verification (2) and the carbonization place cleanup (3) were identified as those that present more postural requirements. These tasks present biomechanical risks in some postural events.

In the task (1) the metallic cylinder must be fulfilled with two wood layers in an organized way, as to insert the bigger amount of wood possible. During this task the cylinder is positioned in a horizontal position.

To execute the task (1) with help of a car, the worker must: charge the car, move the wood pulling the car, and move the wood from the car to the cylinder. The two first steps take from 12 to 16 minutes. In this task we observed that the worker is submitted to a biomechanical effort to collect the wood, when he flexes his spine to lift the piece of wood. In the cylinder charge he also flexes the spine to pick up the piece of wood in the car and with the spine in the vertical position he throws it.

In the task we observed torsion and dorsal flexion of the worker to pick up the piece of wood in the car. The positioning of the wood in the car is done with erected torso and legs.

Moving the wood to the cylinder, the worker pulls the car, what corresponds to an effort of load transport over $20 \mathrm{~kg}$ through a distance of approximately 12 meters. 
To charge the cylinder, there is a worker dorsal motion. With the car in front of the cylinder, the worker transfers the wood organizing it in two levels inside the cylinder. The average time for this task execution is of 4.12 seconds. We may observe that there are postural events with torsion and dorsal flexion together, and elevation of arms over the worker's shoulders.

The analysis based on the OWAS system, indicates that in this task the legs posture is in the category number 2 . The evaluation method prescribes that, even though this does not represent an immediate risk to the workers' health, it must be verified in the next work methods routine revision [5].

In this task the worker torso is erect in $50 \%$ of the time, presents flexion in $25 \%$ and simultaneous flexion and rotation in $25 \%$ of the task execution time. These motions do not require an immediate attention, but the simultaneous dorsal flexion and rotation may cause musculoskeletal lesions in a medium or long time.

The load applied and the arms motions do not require special attention in this task, because they do not represent a biomechanical risk.

To execute the task (1) without car help, we verified that during the morning turn a truck leaves the wood in the carbonization place, in front of the cylinder to be charged. Thus, the workers take the wood and put them in the cylinder.

The sequence of motions in this task has an average execution time of 9.7 seconds and occurs with dorsal flexion and torsion, and also with arms lifted up to the shoulders height with less than $20 \mathrm{Kg}$.

In the research we also observed that the worker walks to the place where the pieces of wood where left, flexes his spine to take the wood in the ground, and loads to the cylinder, putting the wood inside it, with an average execution time of 9.7 seconds.

It was verified that the positioning of the wood in the cylinder requires different worker postures. This varies with the height of the wood layer in the cylinder. Each cylinder takes two wood layers. The individual weight of each piece of wood is less than $15 \mathrm{~kg}$.

It was observed that as the worker is putting the pieces of wood in the cylinder, the spine flexion angle varies, until it reaches the musculoskeletal elongation to execute the task when greater height is required.

Analyzing the motions in the task (1) without the help of car we observed that the torso in flexion and rotation and the legs stopping standing are in category 3 , and deserves attention because may cause lesion musculoskeletal in short time [5]. This evaluation results from the relation between the postures and permanence periods that are: $67 \%$ with erect torso and $33 \%$ with torso in flexion and rotation; $67 \%$ with arms below the shoulders and $33 \%$ with the arms above the shoulders; $33 \%$ of time standing up supported by the two legs, $33 \%$ with the bending knees and walking in $33 \%$ of the time. It was considered in all analyses a load less than $20 \mathrm{~kg}$.

This task may be subdivided into three sub-tasks: take the piece of wood; transport the wood to the cylinder; place the wood inside the cylinder.

The complete motion average time is of 9.7 seconds and the cylinder charge is repeated with a time interval of 1 hour and 5 minutes, and we verified that around 402 repetitions are necessary to charge each cylinder. As two workers execute the task, we found approximately 200 motion repetitions for each worker per hour.

In the first sub-task, the OWAS analysis verified that the flexion of the knee to pick up the piece of woods is in category 4 . Thus, this posture might negatively affect the task and requires attention because this motion associated to the load has the risk of consecutive musculoskeletal lesions.

The second sub-task presents a posture sequence which, associated to a load below $20 \mathrm{~kg}$, does not represent risk to the task biomechanics.

Accordingly to OWAS system the transportation of wood to the cylinders do not offer immediate risk to the workers' health, as it does not presents leg or torso flexion. Additionally, observed that the posture of legs is in category 2 of motion, and suggest a routine inspection. The other motions do not represent risk of lesion or discomfort.

The sub-task of putting wood inside the cylinder occurs with variation in the worker's posture, according to the height of the stack of pieces of wood. In the highest layers, there's no dorsal flexion, but in the lower levels the worker explicitly flexes the torso to arrange the pieces.

The analysis of this posture via the OWAS method allows us to state that this task can affect the worker's health, and this way, it is in the third category of the system, which demands immediate attention.

By observing the task, we verified that when the worker raises his arms above his shoulders, the stacking demands an elongated posture, with no flexion of torso or legs. However, the position of the arms, above the shoulders, requires immediate attention. The erected torso and the adopted load are elements that do not represent risk to the worker's health, but 
the position of legs is in category 2 and the arms above the shoulders are in category 3 .

We observe that while the sub-tasks of transporting and loading the wood into the cylinder do not offer biomechanical risks. The postures adopted for picking the wood are in category 4 , which, according to the OWAS analysis, deserves immediate attention because it offers imminent risk of lesions. This classification is due to the flexion and rotation of the torso associated to the flexion of the knees in the standing up position.

In the task of loading the cylinder, a worker picks a piece of wood and takes it to the cylinder. There, a second worker receives it and puts it inside the cylinder, as shown in figure 29.

The sequence of motions in this task involves postures already evaluated in this study. As both workers perform their activities alternately, we considered the task as a single sequence in the analysis.

The forth team member divides his work into several tasks, which present variation between physical and mental demands. During this research, we observed that, among his tasks, e evaluation of carbonization involves postures subject to musculoskeletal constraint risks.

In the activity of verification of carbonization, a worker visually verifies the status of wood burning which keeps the heating of the cylinder during carbonization and moves the ignition ember.

The task consists in observing through windows of approximately $0.50 \mathrm{~m} \times 0.30 \mathrm{~m}$, located in the floor of the burn place, and inserting a tool through this window to move the ember. The worker walks the entire perimeter of the carbonization chamber and flexes the body to see through the windows located in each of the four chamber walls.

Each chamber is verified once per carbonization hour. We observed that the complete verification procedure takes about 01 minute and 16.6 seconds and in this task the worker assumes several postures shown in figure 30 .

The height of the windows makes the access of the tool to the ember more difficult, as well as the observation by the worker. While verifying, he stays in with flexed torso and legs, making arm motions that cause horizontal motions of the tool.

In the application of the Corlett and Manenica Diagram, the worker did not mention pains or musculoskeletal discomfort. However, the results of the macroscopic observation pointed to biomechanical risks in the execution of these tasks. According to the OWAS system, the sequence of postures adopted by the worker when verifying the chambers present the following results: the event related to the legs postures in motion during $33 \%$ of the total time (standing up with flexed knees) is classified as category 3; the posture on the knees in $33 \%$ of the motion, as category 2 ; and the dorsal flexion in $33 \%$ of the time was inserted in category 2 . Torso and arms postures and the applied load do not represent considerable biomechanical effort.

The sub-task of opening the window presents a apparently uncomfortable posture, but when applying the Corlett and Manenica Diagram, the worker did not report pain or postural discomfort.

The dorsal flexion in $100 \%$ of the time lies in category 3 , associated to flexion of knees, in category 4 , which deserves immediate attention due to the risks of musculoskeletal lesions [5].

The analysis resulting from the OWAS system demonstrates that the biomechanical risks of the subtasks of opening the window and the actual verification. The inclusion in category 2 suggests the need for attention, due to the risk of musculoskeletal lesion in the long-term.

We observed that the walking does not imply postural risks.

The task of chamber ignition consists in transporting the wood from the yard to the chamber, and there cause the ignition (light the fire). In this operation, approximately $117 \mathrm{Kg}$ of wood are used (approximate average). The motion from collecting the wood to loading it in the chamber takes an average time of 23 minutes.

The motions made by the worker during chamber ignition involve erect torso postures in almost $50 \%$ of the total time, flexed in $25 \%$ and flexion and rotation in $25 \%$. The torso, in this sequence, do not represent immediate postural risk, but the OWAS analysis suggests that the association of dorsal flexion and rotation, as well as the position of legs have attention in the next routine checkup.

The third daily task in the production is the distribution of charcoal in the web of the silos and the removal of the unprocessed charcoal. In this task, the workers use a hoe, which has weight around $8 \mathrm{~kg}$ and the silo is $0.80 \mathrm{~m}$ high above the place floor. Torso and legs apparently do not present any biomechanical demand, contrary to what is observed in the superior limbs.

It is verified in the result of the analysis through the OWAS system that the dorsal flexion in $100 \%$ of the time is the event which causes musculoskeletal constraint, classified as category 3 .

Both characteristics in the motion presented the need for dorsal flexion, being classified in category 3 . 
In one of the postures, the position of the arm above the shoulders height is also inserted in category 3 . The position of legs in both cases lies in category 2 .

It is observed that the individual actions of the sub-tasks are classified as category 2 , which, according to the OWAS system, needs attention in the next routine analysis of the methodology of work, due to the possibility of long-term biomechanical risks.

Analyzing the task of carbonization place cleanup, we verified that the task basically consists in sweeping the charcoal left in the floor, gather it and put in the silos. All workers are in charge of this task, which takes about 15 minutes at the end of each turn. In this task they use sweepers, spades and water jets, besides a transportation handcar.

During the observation, we registered about $6 \mathrm{mi}-$ nutes and 28 seconds in the execution of this task by the worker. The activity in a general way does not represent immediate biomechanical risk, according to the analysis resulting from the OWAS system.

In the analysis of motion in the place cleanup, the OWAS system includes flexion of torso when gathering residuals from the floor in category 2 . The positioning of the arms above the height of shoulders when putting the residuals in containers on the handcar, which happen in $33 \%$ of the time, are also in category 2 and may affect the motion biomechanics in the long-term. The remaining events are evaluated as having no biomechanical risks.

We observed that in the OWAS evaluation, sweeping is not associated with postural risks, but the act of gathering the remainders is in category 2 , suggesting the need for verification in the next routine inspection of the methodology of work.

\subsection{Results by OWAS method classification categories.}

The results of the analysis of the activities are seen in Table 1.

Table1

Evaluation of activities by category

\begin{tabular}{|l|c|c|c|l|}
\hline & Category 1 & Category 2 & Category 3 & Category 4 \\
\hline Torso & $59,7 \%$ & $16,57 \%$ & $23,71 \%$ & \\
\hline Arms & $83,42 \%$ & $16,58 \%$ & & \\
\hline Legs & $33,73 \%$ & $56,73 \%$ & $9,47 \%$ & \\
\hline Load & $100 \%$ & & & \\
\hline
\end{tabular}

The analysis of the postural events demonstrate that in $23.71 \%$ of the activities, the torso presents postures that can be harmful to the workers shortly; the same is applied to the legs is 9.47 of the accomplished tasks.

It is observed that in $16.57 \%$ of the activities the dorsal posture lies in category 2 , as well as the arms in $16.58 \%$ and legs in $56.73 \%$ of the activities. The OWAS System classified category 2 as subject to attention in the next routine inspection, or subject to risks in the long-term.

The events classified in category 1 do not predispose the worker to musculoskeletal lesion risks.

The results presented in Table 1 refer to the analysis of the activities as a whole, relating the posture duration and the time need to execute the activity.

Table 2 presents the evaluation of postures in the sub-tasks in an isolate form and demonstrates that the worker, during the accomplishment of his activities, postures classified by the OWAS System in the four categories, including category 4, subject to immediate risks of musculoskeletal lesions.

Table 2

Activities by category

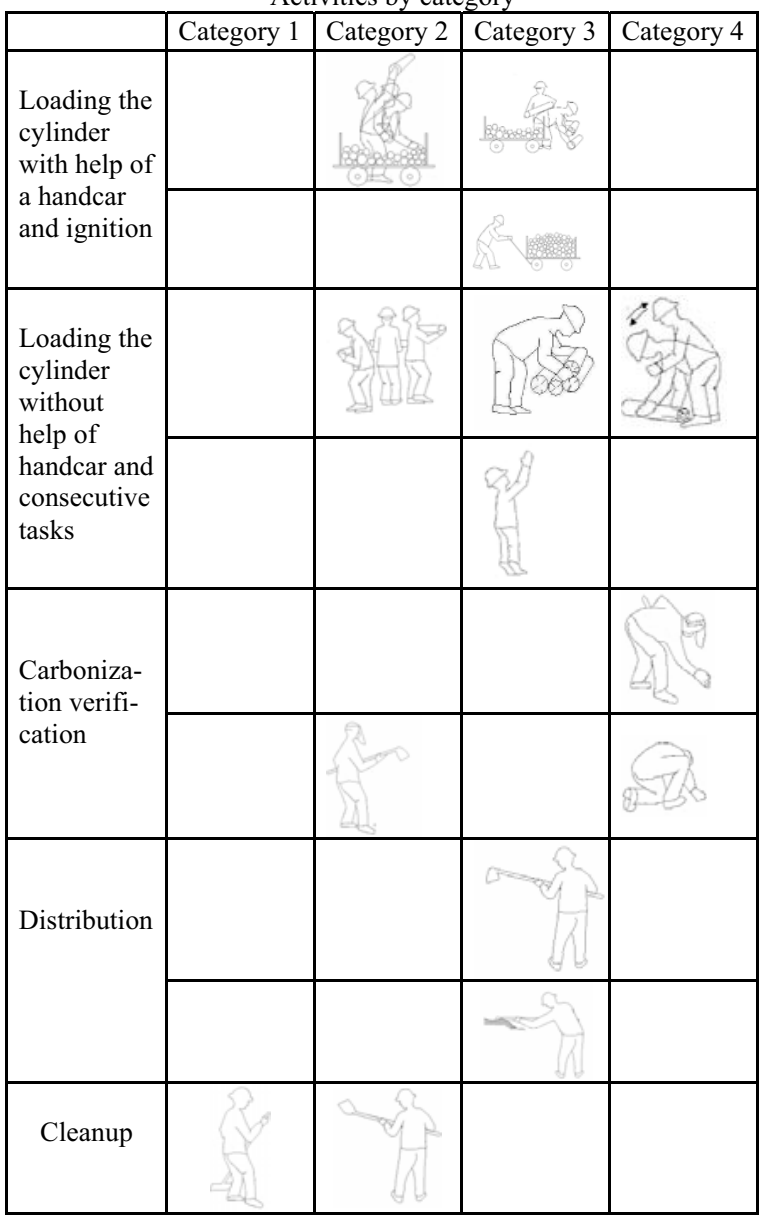


The difference between the evaluation of activated and sub-activities comes from the fact that the OWAS method evaluates the postures adopted in the activities considering the time taken to perform each sub-activity in relation to the time demanded to execute the task as a whole e evaluates the postures in the sub-activities and the events that compose them according to the time of permanence of the worker in the analyzed posture.

\section{Conclusion}

This paper presents an evaluation of postures adopted by workers in the production of charcoal in vertical metallic cylinders, during the execution of their activities.

The 96 postural events analyzed in this study through the OWAS Method consist of 7 activities and 17 sub-activities. The activities are described in Table 4 and from the sub-activities, $12.9 \%$ were classified in category 3 and $4.3 \%$ in category 4 . These postures are characterized by moving the torso in flexion and rotation simultaneously, stand up positioning with flexion of knees and/or postures demanding positioning of arms above the height of shoulders. Category 2 appears in $22.6 \%$ of the events while category 1 appears in $60.2 \%$ of the analyzed postures. The incidence of $39.8 \%$ of postures with some kind of biomechanical stress is an alert for the necessity for attention to the postural conditions to which the workers are submitted.

The result of the application of the Corllet and Maneninca Diagram with all of the population under study, for informing and finding painful areas, indicated that no musculoskeletal pains or lesions were reported, what can be justified by the period of exposition of the workers to these professional activities. The productive method under study is used since October 2006, and there are no records of absences due to musculoskeletal lesions or any complaints from the workers. Also, we observed that the age and fear of losing the job are factors that contribute to the dissonancy between the results achieved by the two methods used in the research.

We observed during the research that the workers are not submitted to load above that determined by Brazilian labor laws, dating from 12/22/1977 and still in force, that stipulate the maximum load of $60 \mathrm{Kg}$ for an male adult employee. The load limit recommended by NIOSH, which establishes the limit of $23 \mathrm{Kg}$ for individual loads lifting is also respected.
Load, then, is not an aggravating factor in the classification of activities and sub-activities by the OWAS method.

We conclude then, in a general manner, that the method for charcoal production based on vertical metallic cylinders partially attends the preservations of occupational health and safety for workers, offering work conditions compatible with the legislation in force. However, the postural aspect is not included in its totality, constituting risks and causing musculoskeletal harms to workers in the short, medium or long-term (depending on the nature of the activity), affecting their health and productivity.

Through this research we noticed that the companies that adopt the model under study can improve the occupational and postural health of the employees, by implementing more expressive practices regarding labor biomechanics, with revisions in the productive method starting from macro-ergonomic focus, such as the restructuration of work places and the adoption of a culture of clarification and dissemination within the organization, about the importance of correct posture for safety and health.

We can also conclude that the Painful Areas Diagram proposed by Corlett and Manenica is not an appropriate research instrument for this study, not just because it approaches a new productive method, but also because the diagram is bases in pre-existing pains and discomforts, showing itself unable to prevent lesions, pains and musculoskeletal discomforts. The OWAS System satisfactorily corresponds to this requisite.

As suggestions for future works we propose the alliance of worker's health in the studied productive method, focusing on other aspects of Ergonomics, approaching the workers' physical performance as much as the cognitive and the development of an instrument that could be used inside the company by workers themselves to identify and correct bad posture during their activities.

\section{References}

[1] Corlett N., Wilson, J. \& Manenica, I. (1986) The ergonomics of working postures. Taylor \& Francis, London.

[2] Dul, J; Weerdmeester, B. Ergonomia Prática. São Paulo: Edgard Blücher, 1995.

[3] Guimarães Neto, R. M. Avaliação técnica e econômica de um forno container em escala industrial. Dissertação de Mestrado. Universidade Federal de Viçosa, MG, 2005.

[4] HignettI, S.; McAatamney, L. Rapid Entire Body Assessment (REBA). Applied Ergonomics, v. 31, p. 201-205, 2000.

[5] Iida, I. Ergonomia, projeto e produção. São Paulo: Edgard Blücher, 2005. 
[6] Karhu, O.; Kansi, P.; Kuorinka, I. Correcting working postures in industry: a practical method for analysis. Applied Ergonomics, v. 8, n. 4, p. 199-201, 1977.

[7] Kroemer, K.; Grandjean, E. Manual de Ergonomia: Adaptando o trabalho ao homem. Porto Alegre: Bookman, 2005. 